\title{
Ongoing Activity Fluctuations in hMT + Bias the Perception of Coherent Visual Motion
}

\author{
Guido Hesselmann, ${ }^{1,2,3}$ Christian A. Kell, ${ }^{4}$ and Andreas Kleinschmidt ${ }^{1,2,3}$ \\ ${ }^{1}$ INSERM, Unité 562, Cognitive Neuroimaging, ${ }^{2}$ Commissariat à l'Energie Atomique, Direction des Sciences du Vivant, Institut d'Imagerie Biomédicale, \\ NeuroSpin, 91191 Gif-sur-Yvette, France, ${ }^{3}$ Université Paris-Sud, 91405 Orsay, France, and ${ }^{4}$ Department of Neurology, Johann Wolfgang Goethe University, \\ D-60325 Frankfurt am Main, Germany
}

\begin{abstract}
We have recently shown that intrinsic fluctuations of ongoing activity during baseline have an impact on perceptual decisions reported for an ambiguous visual stimulus (Hesselmann et al., 2008). To test whether this result generalizes from the visual object domain to other perceptual and neural systems, the current study investigated the effect of ongoing signal fluctuations in motion-sensitive brain regions on the perception of coherent visual motion. We determined motion coherence thresholds individually for each subject using a dynamic random dot display. During functional magnetic resonance imaging (fMRI), brief events of subliminal, supraliminal, and periliminal coherent motion were presented with long and variable interstimulus intervals between them. On each trial, subjects reported whether they had perceived "coherent" or "random" motion, and fMRI signal time courses were analyzed separately as a function of stimulus and percept type. In the right motion-sensitive occipito-temporal cortex (hMT +), coherent percepts of periliminal stimuli yielded a larger stimulus-evoked response than random percepts. Prestimulus baseline activity in this region was also significantly higher in these coherent trials than in random trials. As in our previous study, however, the relation between ongoing and evoked activity was not additive but interacted with perceptual outcome. Our data thus suggest that endogenous fluctuations in baseline activity have a generic effect on subsequent perceptual decisions. Although mainstream analytical techniques used in functional neuroimaging do not capture this nonadditive effect of baseline on evoked response, it is in accord with postulates from theoretical frameworks as, for instance, predictive coding.
\end{abstract}

Key words: predictive coding; perceptual decision; visual awareness; BOLD fMRI; prestimulus activity; consciousness

\section{Introduction}

Fluctuating spontaneous brain activity is a prominent but persistently puzzling feature in any type of neurophysiological recording. It can be thought of as unexplained variance in relation to the experimental paradigm under investigation and has been observed with a wide range of methods on different temporal and spatial scales (Biswal et al., 1995; Kenet et al., 2003; Holcman and Tsodyks, 2006; Fox and Raichle, 2007). In functional magnetic resonance imaging (fMRI) studies, spatially distributed fluctuations of the blood oxygenation level dependent (BOLD) signal have been linked to variability in action [motor output (Fox et al., 2007)] and perception [in the somatosensory and nociceptive domain (Boly et al., 2007)]. We have shown that even local intrinsic activity variations, occurring over and above those that manifest in distributed patterns, play an important role in visual perception. We found that the variability in ongoing BOLD activity of fusiform face-sensitive visual areas (FFA) biases the way in which subjects perceive Rubin's classic "face-vase" ambiguity.

\footnotetext{
Received Sept. 15, 2008; revised Nov. 6, 2008; accepted Nov. 22, 2008.

This work was supported by the Volkswagen Foundation (Germany) and the Agence Nationale de la Recherche (SPONTACT; France). We thank G. M. Kell for ad-hoc technical support.

Correspondence should be addressed to Guido Hesselmann, INSERM Unité 562, NeuroSpin/Commissariat à

I'Energie Atomique, 91191 Gif-sur-Yvette, France. E-mail: g.hesselmann@gmail.com.

D0I:10.1523/JNEUROSCI.4398-08.2008

Copyright $\odot 2008$ Society for Neuroscience $\quad 0270-6474 / 08 / 2814481-05 \$ 15.00 / 0$
}

Right FFA activity levels during prestimulus baseline and at the evoked response peak were significantly correlated with subjects' reports of face percepts (Hesselmann et al., 2008). In line with different theoretical accounts of perceptual decisions (Smith and Ratcliff, 2004; Friston, 2005), however, response peak variability did not originate from a passive propagation of variability before stimulation over time. This latter observation speaks against a simple additive mechanism, by which evoked activity superimposes onto ongoing activity (Arieli et al., 1996).

Here, we sought to investigate whether these effects of ongoing activity fluctuations would also be observed in another domain of vision and, thus, other cortical substrates. We targeted the perception of coherent visual motion and motion-sensitive human brain areas. In the macaque brain, a medial temporal (MT) area is crucial for integrating local motion vectors and, thus, perceiving coherent motion (Born and Bradley, 2005). Direct electrical stimulation of MT biases motion direction judgments (Salzman et al., 1990). A variety of functional neuroimaging studies have related subjects' perceptual experience of motion to activity of hMT +, the assumed human homolog of MT (Goebel et al., 1998; Rees et al., 2000; Serences and Boynton, 2007).

We framed our experiment as a perceptual decision task on an ambiguous stimulus. In a sparse event-related fMRI design with long and variable rest intervals, subjects were intermittently exposed to motion stimuli with a coherence level at their previously 


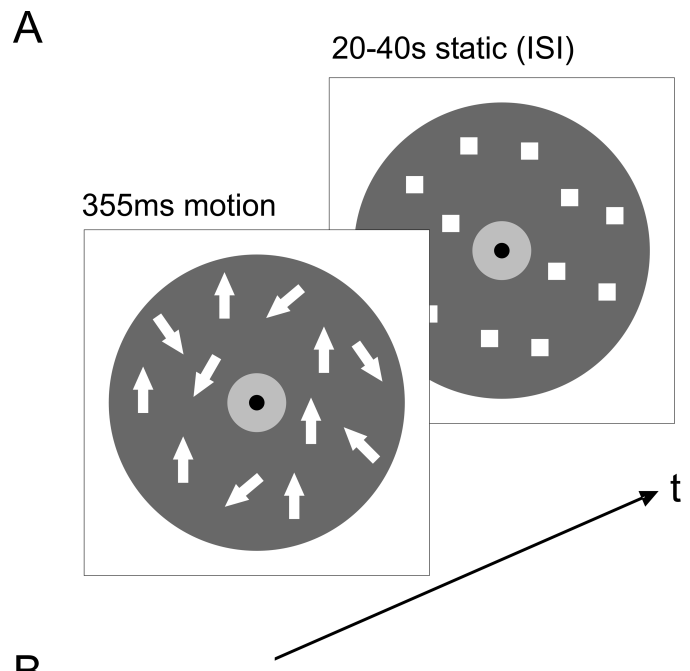

B

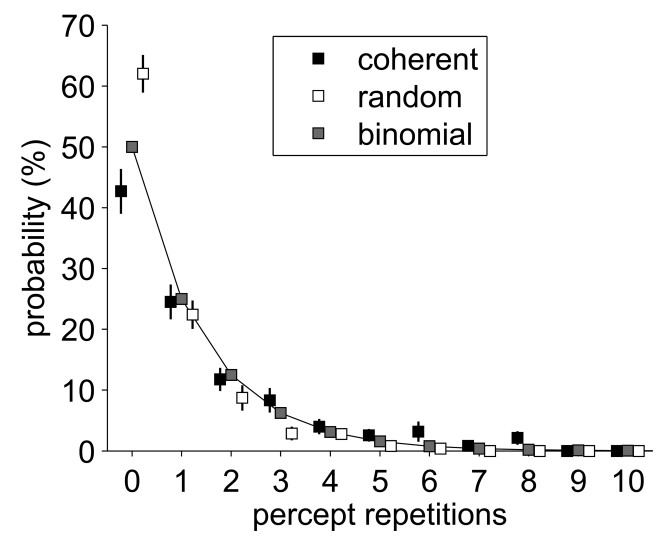

Figure 1. $\quad \boldsymbol{A}, 0$ n every trial, a motion stimulus (either up- or downward) of variable coherence (subthreshold, perithreshold, or suprathreshold) was presented for $355 \mathrm{~ms}$ in a dynamic random dot display. Subjects reported whether they had perceived coherent or random motion. ISIs with stationary display ranged from 20 to $40 \mathrm{~s}$. White arrows represent the motion vectors of dynamic dots. $\boldsymbol{B}$, The incidence of repetitions for either percept averaged across all ISIs can very well be approximated by a binomial distribution (goodness-of-fit $R^{2}=0.98$, for coherent percepts; $R^{2}=0.91$, for random percepts).

determined detection thresholds as well as to occasional subliminal or supraliminal stimuli. Subjects reported for each trial whether they perceived coherent or random motion. We tested whether higher prestimulus BOLD activity levels in hMT + biased perception toward reporting coherent visual motion. Furthermore, we analyzed the effect of prestimulus activity on the peak BOLD response to corroborate, or not, our earlier finding of a nonadditive relation between evoked and ongoing activity.

\section{Materials and Methods}

Subjects and experimental protocol. Twelve right-handed subjects with normal or corrected-to-normal visual acuity (six female, average age: 21 years, range: $19-30$ years) gave written informed consent. We had ethics committee approval for this study. Stimuli were video-projected at a 120 $\mathrm{cm}$ viewing distance using a VSG2/5 stimulus generator card (Cambridge Research Systems). Stimuli were dynamic dot displays of 500 white squares $\left(\right.$ size $0.2^{\circ}$ ) randomly distributed on a dark gray annulus $\left(23^{\circ}\right)$ (Fig. $1 \mathrm{~A}$ ). Subjects were instructed to maintain gaze within a central blue rectangle $\left(1^{\circ}\right)$ surrounded by a light gray circular patch $\left(3^{\circ}\right)$ throughout the experimental sessions. Eye-tracking during fMRI was not available, but off-line recordings ensured that subjects could well comply with this instruction. For $355 \mathrm{~ms}$ intervals, stimuli moved up- or downward, at $14 \%$, and with variable coherence. Noise dots moved in a "random walk." Signal dots had a limited lifetime of one frame (17 ms). Subjects were asked to report as quickly and accurately as possible by hand key presses after each stimulus whether they had perceived coherent or random motion.

Before scanning we used the method of constant stimuli (30 trials each with motion coherence of $2,6,10,14,18,22$, and $26 \%$, order randomized, no feedback) to determine individual motion coherence thresholds (50\% level of a cumulative normal distribution fit, average motion coherence threshold across subjects $13 \%$, range $8-20 \%$ ). During fMRI, three motion coherence levels were used: subliminal ( $1 \%$ coherence, 20 trials), periliminal (individually estimated threshold, 60 trials), and supraliminal (30\% coherence, 20 trials). Stimuli were presented in two 25 min sessions with 50 trials each. Between stimuli, the display was static for interstimulus intervals (ISI) of 20 to $40 \mathrm{~s}$ that were randomly selected from a uniform distribution.

Acquisition and processing of $f M R I$ data. Functional images for two 1000 volume experimental sessions and a 208 volume localizer session were acquired on a 3T MRI scanner (Tim Trio, Siemens) by T2*weighted gradient-echo echo-planar imaging [ 25 slices, repetition time $(\mathrm{TR})=1500 \mathrm{~ms}$, echo time $(\mathrm{TE})=30 \mathrm{~ms}$, voxel size $3 \times 3 \times 3 \mathrm{~mm}$, interslice gap 20\%]. Anatomical images were acquired with a T1weighted MPRAGE sequence $(160$ slices, $\mathrm{TR}=2300 \mathrm{~ms}, \mathrm{TE}=2.98 \mathrm{~ms}$, field of view 256, voxel size $1.0 \times 1.0 \times 1.1 \mathrm{~mm}$ ). We used SPM5 (http:// www.fil.ion.ucl.ac.uk, Wellcome Trust Centre for Neuroimaging, London, UK) for image preprocessing (realignment, coregistration, normalization to Montréal Neurological Institute stereotactic space, spatial smoothing with an isotropic Gaussian kernel of 6 and $12 \mathrm{~mm}$ full-widthhalf-maximum for single subject and group analyses, respectively) and estimation of the statistical maps.

Definition of regions of interest. Localizer fMRI sessions identified cortical regions sensitive to two types of coherent visual motion, up- or downward motion and an expanding "starfield." Continuous $16 \mathrm{~s}$ motion blocks were separated by $10 \mathrm{~s}$ stationary periods, and each condition was repeated over six blocks in counter-balanced order. Motionsensitive areas were identified by mapping for each subject the contrast "motion $>$ stationary" at $p<0.001$, uncorrected. A local maximum near the ascending limb of the inferior temporal sulcus was defined as hMT + , a second posterior, superior, and medial maximum putatively labeled as hV3/V3A [(Tootell et al., 1997) see supplemental Table T1, available at www.jneurosci.org as supplemental material for coordinates of all regions of interest (ROIs)].

Analysis of $F M R I$ data. After removing session effects and linear trends from the BOLD signal time series, we extracted for each ROI the percentage signal change time courses of all periliminal trials from 4 scans $(6 s)$ before to 12 scans $(18 \mathrm{~s}$ ) after target onset and sorted them according to coherent and random percepts. Based on our previous findings (Hesselmann et al., 2008), three time points were chosen for statistical analysis: time points -1.5 and $0 \mathrm{~s}$ in the immediate prestimulus baseline as well as the peak hemodynamic response at $6 \mathrm{~s}$. Data were submitted to a repeated-measures $2 \times 3$ ANOVA ("percept" $\times$ "time point") and post hoc testing with paired $t$ tests.

\section{Results}

\section{Behavioral data}

Periliminal motion stimuli yielded across subjects 57\% coherent and $43 \%$ random percepts $\left[t_{(11)}=2.06\right.$, not significant (n.s.); range for coherent $43-77 \%$ ]. This ratio was consistent across the two sessions. Subliminal stimuli were more often seen as random than periliminal stimuli $\left(74 \%, t_{(11)}=5.64, p<0.001\right)$ and supraliminal stimuli more often as coherent $\left(94 \%, t_{(11)}=17.97\right.$, $p<0.001)$. We found no carry-over of percepts reported in successive trials, with average incidence of percept repetitions (across trials and coherence levels) well approximated by a binomial distribution indicating stochastic behavioral reports (Fig. $1 B)$. Subjects responded faster in supraliminal $(1119 \mathrm{~ms})$ than subliminal ( $1259 \mathrm{~ms}$ ) and periliminal trials ( $1241 \mathrm{~ms})$. In periliminal trials, subjects responded $\sim 160 \mathrm{~ms}$ faster for coherent $(1160 \mathrm{~ms})$ than for random percepts $\left(1324 \mathrm{~ms}, t_{(11)}=4.03, p<0.01\right)$. 

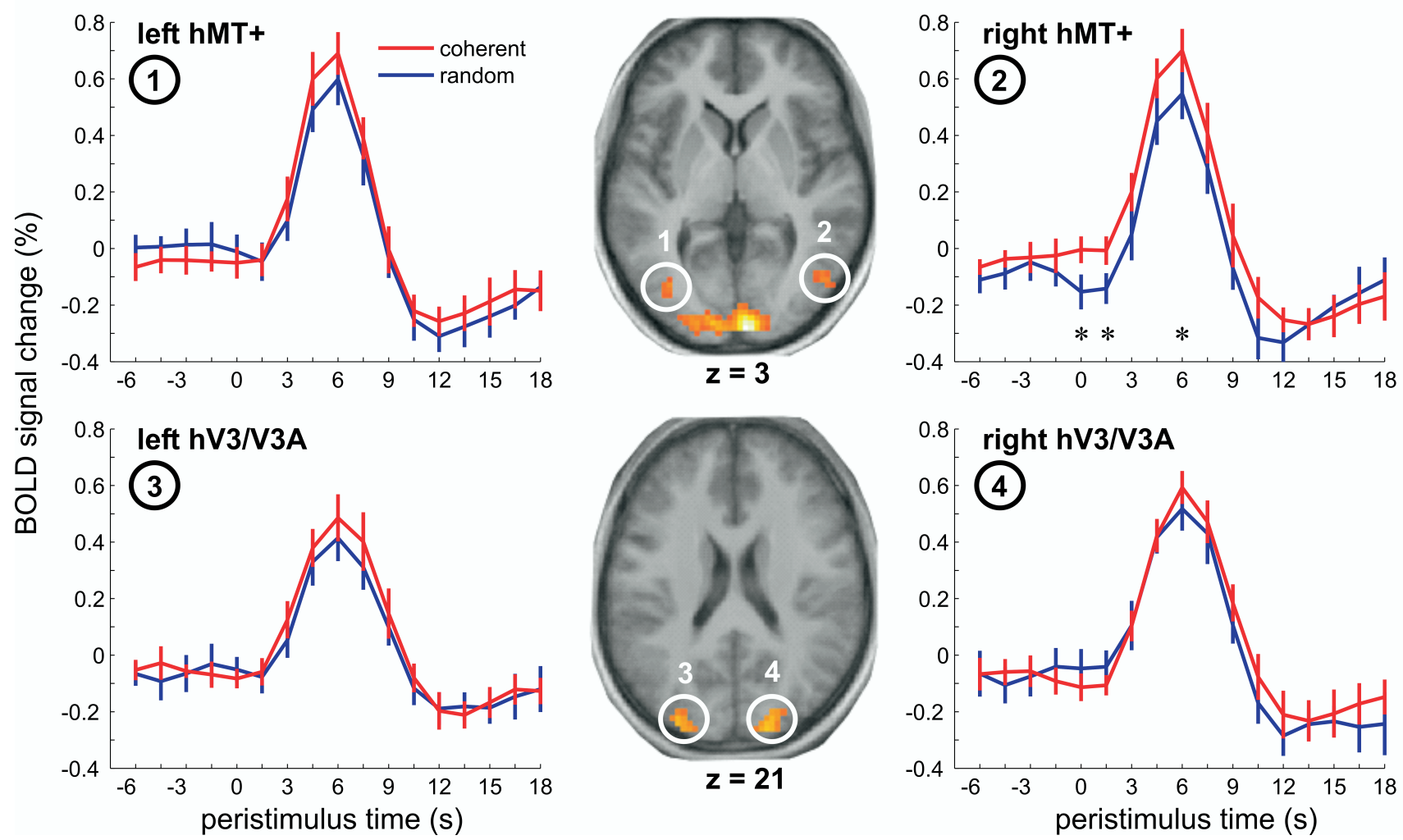

Figure 2. Peristimulus fMRI signal time courses from motion-sensitive brain regions, hMT + (top) and putative hV3/V3A (bottom). Data averaged across all subjects with error bars representing SE and filtered with a [ 121 l] kernel for display purposes. Statistical parametric maps from an individual subject localize motion areas as identified by the contrast "motion $>$ stationary" ( $p<$ $5^{*} 10^{-5}$, uncorrected, axial slices overlaid on the average anatomy).

\section{Functional imaging data}

All motion-sensitive ROIs showed a clear hemodynamic response in the periliminal experimental trials of interest. A significantly larger response for coherent than for random perception of periliminal stimuli was found only in right hMT + (main effect "percept," $F_{(1,11)}=8.91, p=0.012$, post hoc $t_{(11)}=2.26, p=$ 0.045 ) but not in left hMT + and both hV3/V3A (Fig. 2). Significant percept-dependent signal differences in prestimulus activity during these trials were also restricted to right $\mathrm{hMT}+$. In coherent trials, activity starting at $\sim 1.5 \mathrm{~s}$ before stimulus onset was higher than in random trials. Planned post hoc tests revealed an effect at the immediate prestimulus time point $0 \mathrm{~s}\left(t_{(11)}=3.61\right.$, $p<0.005)$. The activity difference at $-1.5 \mathrm{~s}$ did not reach significance, whereas exploratory analysis of the peristimulus time point $1.5 \mathrm{~s} \operatorname{did}\left(t_{(11)}=2.25, p=0.046\right)$, but this time segment was not targeted a priori, and the effect did not survive Bonferroni correction for testing at multiple time points. Overall, the effect could neither be related to a significant activity increase over time in one nor a decrease within another of the two conditions.

To explore the spatial specificity of the prestimulus signal for perceptual performance, we analyzed time courses in a set of control regions that significantly activated or deactivated during perceptual decisions (supplemental Fig. S1, available at www. jneurosci.org as supplemental material). These regions included areas involved in early visual motion processing (V1/V2), as well as attention and perceptual decision making (right inferior parietal lobule, right and left frontal eye field, right inferior frontal gyrus, and anterior cingulate cortex). No region showed perceptdependent signal differences in the prestimulus baseline epoch, and additional voxel-based whole brain analyses were also negative. Early visual cortex showed a significantly larger evoked re- sponse in random than in coherent trials when testing for the effect of condition in an ANOVA $\left(F_{(1,11)}=7.391, p=0.020\right)$. Although this difference appears to arise at time points that cannot yet carry stimulus-driven signal, this observation of opposite sign as in hMT + remained nonsignificant for individual time points including baseline.

To further probe the relationship between right hMT + activity and perception, we analyzed choice probabilities for the immediate prestimulus baseline $(0 \mathrm{~s})$ and the peak response $(6 \mathrm{~s})$. Across subjects, a coherent percept could be predicted significantly better than chance from the prestimulus baseline level $\left[\right.$ mean area under the curve $(\mathrm{AUC})=0.57, t_{(11)}=3.89, p<$ 0.005 ) as well as from the response peak (mean AUC $=0.55, t_{(11)}$ $=2.02, p=0.035)$. An earlier reference time point at $-6 \mathrm{~s}$ was of no predictive power (mean AUC $=0.50$ ), underlining the temporal specificity of the prestimulus effect in right hMT + and hence activity fluctuations as its origin (supplemental Fig. S2, available at www.jneurosci.org as supplemental material). Interestingly, however, the intermediate time points between the prestimulus baseline segment and the response peak also failed to show significant prediction. This observation suggests that the fMRI signal carries two different types of information that are both related to perception but in a different way. One source of information can be captured before the effects of sensory stimulation, the other in their presence, but at time points mixing the two, these effects cancel out. In line with this interpretation, we observed that classification either from the prestimulus period or from the response peak was preserved when testing signal at each time point after its covariance with signal at the other time point had been removed by regression.

Next, we analyzed behavioral correlations with activity levels 
at baseline and response peak in periliminal trials. Although on average RTs differed between the two percepts, they did not within or across conditions correlate trial-by-trial with activity levels in right $\mathrm{hMT}+$, neither at the prestimulus baseline, nor at the peak response (for findings in other regions, see supplemental Fig. S1, available at www.jneurosci.org as supplemental material). This lack of reaction time correlation is compatible with a taskunrelated origin of prestimulus signal variations and is often considered a characteristic of "stimulus-independent" as opposed to "stimulus-oriented" neural activity (Gilbert et al., 2007). RT shortening is generally considered a hallmark of deploying attention, and the variability in baseline therefore cannot be linked to attentional mechanisms in a straightforward way.

Finally, we assessed the trial-by-trial variability in ongoing and evoked activity and their relation to perceptual outcome of periliminal trials. If the fundamental mechanism that links evoked responses to ongoing activity was additive, as suggested by earlier work in anesthetized animals (Arieli et al., 1996), then there should be a positive correlation across trials between activity levels at the response peak with those during the preceding baseline. In other words, the higher activity before stimulation, the higher the peak of the response amplitude. We hence calculated the linear regression of the peak response at $6 \mathrm{~s}$ on the prestimulus activity at $-1.5 \mathrm{~s}$ for each subject (Fig. $3 A$ ). We then submitted the resulting $\beta$ values to a paired test. In random trials, there was indeed a weak positive correlation between ongoing and evoked activity levels but not in coherent trials (Fig. 3B). Accordingly, statistical testing showed a significant interaction of these correlations with perceptual outcome. This interaction expresses that hMT + response peaks were significantly less correlated with ongoing activity when subjects perceived coherent motion than when they failed to do so. This observation is not trivial because on average both ongoing and evoked hMT + activity were correlated with coherence perception in the identical stimulus. Yet, notwithstanding this average result, the prestimulus and the stimulus-driven activity were not on a trial-by-trial basis correlated with each other, which in turn suggests that variability in both time segments contributes independently to whether coherent motion is perceived on individual periliminal trials (Smith and Ratcliff, 2004).

\section{Discussion}

In the present study, we extend our earlier observation (Hesselmann et al., 2008) to a different domain of visual perception and thus corroborate an effect that we propose to be a general principle. We show that local ongoing activity in motion-sensitive area $\mathrm{hMT}+$ significantly predicted whether for a periliminal random dot motion stimulus coherence was perceived or not. We expected this region to be the most suitable candidate for detecting an effect from ongoing activity on perception, because a wide functional neuroimaging literature has shown responses in this area to correlate with visual motion perception. Recent multivoxel response pattern analyses, for instance, have shown that fMRI activation in hMT + matches the observer's perception, whereas responses in other visual areas do not (Serences and Boynton, 2007).

We did not observe effects from ongoing activity in any other

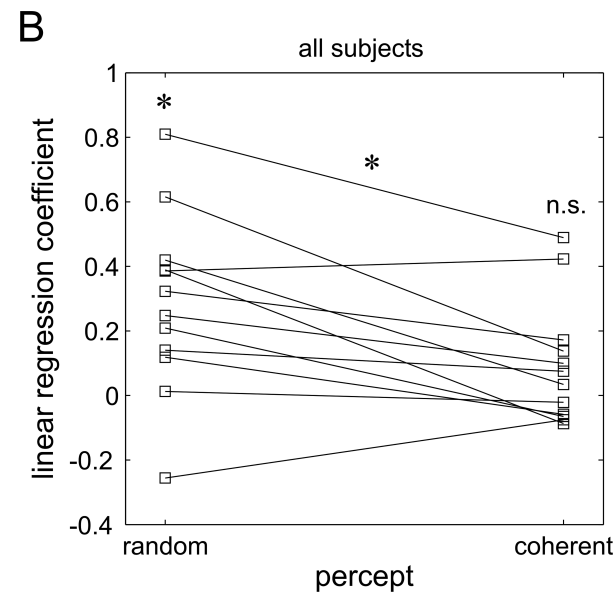

single subject

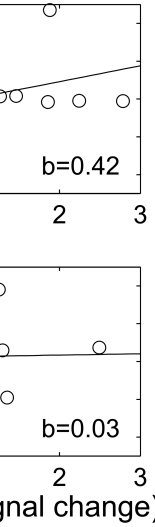

Figure 3. Single subject and mean percept-dependent regressions between trial-by-trial prestimulus activity at $-1.5 \mathrm{~s}$ and peak activity at $6 \mathrm{~s}$ in right hMT.$+ A$, Illustrated for a single subject, linear regressions ( $y=a+b x+c$ ) were fitted to the data (top for trials with random percepts, bottom for coherent percepts). $\boldsymbol{B}$, Plot of the linear regression ficients $b$ for all subjects as a function of percept. As indicated by asterisks, coefficients were significantly larger than 0 in random trials $\left(0.28, t_{(11)}=3.55, p<0.01\right)$ but not in coherent trials $\left(0.09, t_{(11)}=1.7, n . s.\right)$, and significantly different between

brain regions responding to the task, which probably reflects the fact that our paradigm was optimized to target functional properties of hMT+ (Shadlen and Newsome, 2001). An important difference of our previous and current findings from spontaneous activity fluctuations compared with artificial activity manipulation by microstimulation of specialized cortical areas is that microstimulation before stimulus onset has to our knowledge not yet been shown to influence perceptual outcome (Salzman et al., 1990). We propose that this difference between experimental methods can be explained by the nonphysiological structure of microstimulation-induced activity changes as opposed to the physiological nature of spontaneous BOLD fluctuations.

That our effect was confined to right hMT + is internally consistent within our data set in that this region was also the only one to demonstrate a significant response difference to the periliminal stimulus as a function of how it was perceived. In other words, this region seems to be the most sensitive one in relation to taskrelevant stimulus features. This interpretation is congruent with a larger literature which has shown a right hemisphere lateralization of hMT + activity when wide-field optic flow stimuli are used, as was the case here including our masking of the central field portion (Peuskens et al., 2001).

Previous experiments in anesthetized animals described that the stimulus-evoked response simply adds to the level of ongoing activity present during stimulus onset (Arieli et al., 1996). This additive mechanism could nonetheless be functionally relevant, for instance by determining whether a neural response on a given trial passes a threshold. We found that in awake humans the impact of local spontaneous ongoing activity variations on stimulus-evoked activity is not simply additive but interacts with perceptual outcome. Our findings, including lower evoked response in V1 for coherent trials, are compatible with theoretical perspectives that emphasize the constructive nature of perceptual processes, e.g., predictive coding and related conceptual frameworks (Friston, 2005; Harrison et al., 2007). Seen from this perspective, it could be argued that spontaneous activity fluctuations carry "dynamic predictions," as suggested recently (Fox and Raichle, 2007).

Dynamic predictions could manifest in prestimulus activity as a prior against which the incoming sensory input is matched. With independent variability in both parameters, the prior and 
the input representation (Philiastides et al., 2006), one can sketch four illustrative situations. High prior activity and congruent sensory input processing generate little mismatch or prediction error and thus a weak evoked response in a coherent perception. Low prior activity but strongly represented coherence in the input signal generates a mismatch with a strong evoked response and the perception of coherence. This would explain why response peak activity levels in coherent percepts do not increase with baseline activity. Conversely, if the prior is high but the trial is perceived as random, there has been a fair amount of prediction error, and thus a greater signal increment than in trials in which the prior was low, and which were reported as random and thus congruent with the prior. The latter two cases would then explain why in the case of random percepts there is a weak but significant positive relation between ongoing and evoked activity levels. Importantly, the prestimulus prior itself also represents a prediction error, namely between the top-down prediction of a specific sensory signal and the prestimulus instance when such input is still lacking.

Beyond such a speculative account, however, the key point is that the relation between ongoing and evoked activity depends on perceptual outcome. This observation itself cannot be explained in a simple mechanistic model in which responses add onto ongoing activity levels, and where this relation should be the same across trials whatever the perceptual outcome. As in our previous experiment, the functionally significant effect was apparently restricted to a focal brain region that is crucial for the task at hand. Our findings characterize the consequences of such spontaneous variations in ongoing activity for visual motion perception and thus inform models of perceptual decision-making (Heekeren et al., 2008).

The physiological origin or cognitive meaning of spontaneous fluctuations in the awake brain are not yet fully understood and cannot be clarified by our or any related results, because studies that address functional significance inevitably require an overarching task context, so as to probe the perceptual or behavioral consequences of baseline signal fluctuations. In current views on the nature of spontaneous brain activity (Fox and Raichle, 2007), signal fluctuations observed in fMRI may span processes at several different levels of neural activity, ranging all the way from intrinsic noise over low-level physiological processes to uncontrolled mental activity with varying degrees of contribution from the actual experimental context. One of several important unresolved issues is the relation of baseline fluctuations to attention (Boly et al., 2008). Across a distributed system of areas underpinning selective attention, variability in cued prestimulus fMRI signal changes has been found to predict perceptual performance (Sapir et al., 2005). Allocation of attention raises the baseline fMRI signal even in the absence of sensory input (Kastner et al., 1999) but also leads to RT shortening and an often linear increase in evoked sensory responses (Chawla et al., 1999; McMains et al., 2007). That the effects in our study did not show these but in part opposite properties cannot formally rule out a contribution from attentional mechanisms but renders them unlikely. In the absence of a full account of origin and connotation of spontaneous activity, however, our findings are important because they show that this unexplained variance, whatever its origin, contributes significantly to the way in which the brain and the observer respond to external sensory stimuli (Deco and Romo, 2008).

\section{References}

Arieli A, Sterkin A, Grinvald A, Aertsen A (1996) Dynamics of ongoing activity: explanation of the large variability in evoked cortical responses. Science 273:1868-1871.

Biswal B, Yetkin FZ, Haughton VM, Hyde JS (1995) Functional connectiv- ity in the motor cortex of resting human brain using echoplanar MRI. Magn Reson Med 34:537-541.

Boly M, Balteau E, Schnakers C, Degueldre C, Moonen G, Luxen A, Phillips C, Peigneux P, Maquet P, Laureys S (2007) Baseline brain activity fluctuations predict somatosensory perception in humans. Proc Natl Acad Sci U S A 104:12187-12192.

Boly M, Phillips C, Balteau E, Schnakers C, Degueldre C, Moonen G, Luxen A, Peigneux P, Faymonville ME, Maquet P, Laureys S (2008) Consciousness and cerebral baseline activity fluctuations. Hum Brain Mapp 29:868-874.

Born RT, Bradley DC (2005) Structure and function of visual area MT. Annu Rev Neurosci 28:157-189.

Chawla D, Rees G, Friston KJ (1999) The physiological basis of attentional modulation in extrastriate visual areas. Nat Neurosci 2:671-676.

Deco G, Romo R (2008) The role of fluctuations in perception. Trends in Neurosciences 31:591-598.

Fox MD, Raichle ME (2007) Spontaneous fluctuations in brain activity observed with functional magnetic resonance imaging. Nat Rev Neurosci 8:700-711.

Fox MD, Snyder AZ, Vincent JL, Raichle ME (2007) Intrinsic fluctuations within cortical systems account for intertrial variability in human behavior. Neuron 56:171-184.

Friston KJ (2005) A theory of cortical responses. Philos Trans R Soc Lond B Biol Sci 360:815-836.

Gilbert SJ, Dumontheil I, Simons JS, Frith CD, Burgess PW (2007) Comment on "Wandering minds: the default network and stimulusindependent thought". Science 317:43.

Goebel R, Khorram-Sefat D, Muckli L, Hacker H, Singer W (1998) The constructive nature of vision: direct evidence from functional magnetic resonance imaging studies of apparent motion and motion imagery. Eur J Neurosci 10:1563-1573.

Harrison LM, Stephan KE, Rees G, Friston KJ (2007) Extra-classical receptive field effects measured in striate cortex with fMRI. Neuroimage 34:1199-1208.

Heekeren HR, Marrett S, Ungerleider LG (2008) The neural systems that mediate human perceptual decision making. Nat Rev Neurosci 9:467-479.

Hesselmann G, Kell CA, Eger E, Kleinschmidt A (2008) Spontaneous local variations in ongoing neural activity bias perceptual decisions. Proc Natl Acad Sci U S A 105:10984-10989.

Holcman D, Tsodyks M (2006) The emergence of up and down states in cortical networks. PLOS Computational Biology 2:174-181.

Kastner S, Pinsk MA, De Weerd P, Desimone R, Ungerleider LG (1999) Increased activity in human visual cortex during directed attention in the absence of visual stimulation. Neuron 22:751-761.

Kenet T, Bibitchkov D, Tsodyks M, Grinvald A, Arieli A (2003) Spontaneously emerging cortical representations of visual attributes. Nature 425:954-956.

McMains SA, Fehd HM, Emmanouil TA, Kastner S (2007) Mechanisms of feature- and space-based attention: response modulation and baseline increases. J Neurophysiol 98:2110-2121.

Peuskens H, Sunaert S, Dupont P, Van Hecke P, Orban GA (2001) Human brain regions involved in heading estimation. J Neurosci 21:2451-2461.

Philiastides MG, Ratcliff R, Sajda P (2006) Neural representation of task difficulty and decision making during perceptual categorization: a timing diagram. J Neurosci 26:8965-8975.

Rees G, Friston K, Koch C (2000) A direct quantitative relationship between the functional properties of human and macaque V5. Nat Neurosci 3:716-723.

Salzman CD, Britten KH, Newsome WT (1990) Cortical microstimulation influences perceptual judgments of motion direction. Nature 346:174-177.

Sapir A, d'Avossa G, McAvoy M, Shulman GL, Corbetta M (2005) Brain signals for spatial attention predict performance in a motion discrimination task. Proc Natl Acad Sci U S A 102:17810-17815.

Serences JT, Boynton GM (2007) The representation of behavioral choice for motion in human visual cortex. J Neurosci 27:12893-12899.

Shadlen MN, Newsome WT (2001) Neural basis of a perceptual decision in the parietal cortex (area LIP) of the rhesus monkey. J Neurophysiol 86:1916-1936.

Smith PL, Ratcliff R (2004) Psychology and neurobiology of simple decisions. Trends Neurosci 27:161-168.

Tootell RB, Mendola JD, Hadjikhani NK, Ledden PJ, Liu AK, Reppas JB, Sereno MI, Dale AM (1997) Functional analysis of V3A and related areas in human visual cortex. J Neurosci 17:7060-7078. 